\title{
Assessment of hematological bio markers during vaccination and challenge of brucella melitensisin
} goats

\begin{abstract}
Brucella melitensis affects both animals and humans and causes severe economic losses mainly due to abortions, stillbirths and reproductive disorders in animals besides being potentially hazardous to the animal handlers. The losses can be prevented and controlled through the use of Rev.1 vaccine. The protective ability of Rev.1 against locally prevalent virulent strain and its ability to induce adaptive immune response involve cellular interactions depending upon patho-physiological changes and induction of immune response. Thus to assess the changes in haematological parameters like number and types of circulating cells in blood, pure bred Brucella free native Jamunapari adult goats were vaccinated with Rev.1 and challenged $28^{\text {th }}$ day post vaccination with locally prevalent virulent Brucella melitensis biovar3 Indian isolate. The blood samples were collected from healthy, vaccinated and challenged goats at different intervals and subjected to assess fourteen different haematological parameters till $90^{\text {th }}$ day post challenge. The application of Rev.1 vaccine in pure bred Jamunapari goats did not adversely influenced majority of blood parameters. Moreover, the percentage of lymphocytes, granulocytes and monocytes in total white blood cell count during vaccination with live Rev.1 vaccine does not have any conclusive assessment. Challenge with live virulent culture increased granulocytes initially but later on lymphocytes number increased significantly. The number of monocytes, the cells mainly involved in brucella clearance from infected cells increased initially after challenge but overall cell percentage remained almost unaltered. Out of fourteen blood parameters under study, it is only the total count of lymphocytes and granulocytes that can be used as haematological biomarkers to draw some inference regarding the status of infection in vaccinated animals.
\end{abstract}

Keywords: brucella melitensis, haematology, rev.1, vaccination, challenge, goat
Volume 4 Issue 2 - 2017

\author{
Amit Kumar, 'VK Gupta, ${ }^{2}$ AK Verma, ${ }^{3}$ Ashok \\ Kumar, ${ }^{4}$ SK Yadav' \\ 'Department of Veterinary Microbiology, College of Veterinary \\ Sciences, DUVASU,India \\ 2Indian Veterinary Research Institute (IVRI), India \\ ${ }^{3}$ Department of Veterinary Epidemiology and Preventive \\ Medicine, College of Veterinary DUVASU,India \\ ${ }^{4}$ Division of Goat Health, Central Institute for Research on \\ Goats (CIRG), India
}

Correspondence: Amit Kumar, Assistant Professor,

Department of Veterinary Microbiology, College of Veterinary Sciences, DUVASU, Mathura-28I00I, India, Tel +919412120813, Email balyan74@gmail.com

Received: February 02, 2017 | Published: March 30, 2017

\section{Introduction}

Brucellosis, particularly due to Brucella melitensis is a well established world wide zoonotic disease. It affects both animals and humans and causes severe economic losses worldwide including India. ${ }^{1,2}$ These economic losses are mainly due to abortions, stillbirths and reproductive disorders in animals besides being potentially hazardous to the animal handlers. Infected parturitions (normal birth or abortion) and infected males play an important role in the spread of infection in herds. ${ }^{2}$ The $13.4 \%$ of kid's loss have been reported owing to abortions and stillbirth in semi-intensively managed goat herds in India. ${ }^{3}$ The control of $B$. melitensis infection is necessary to reduce livestock economic losses as well as to avoid zoonotic transfer. Thushuman losses in the form of lost man days and medical consequences in human can be prevented and controlled through the use of vaccines. ${ }^{1}$ Presently available and well established vaccine Rev.1, a live $B$. melitensis vaccine, is the most widely used vaccine in control programmes against brucellosis in small ruminants all over the world. ${ }^{4}$ However, Rev. 1 vaccine shows a considerable degree of virulence and induces abortions when the first vaccine dose is administered during pregnancy. The full dose vaccine also leads to release of Brucella in milk that can be the source of human transmission. ${ }^{4,5}$ Due to these constraints Rev.1 has not been accepted in India for prevention/ control or eradication of brucellosis in sheep and goats. Moreover, protective ability against locally prevalent virulent strain and its ability to induce adaptive immune response in native breed are always under question as protective efficacy of Rev.1 vaccine greatly depends on the Brucella virulence and challenge dose presented to host species. The genetic makeup of host also affects the effectiveness and protective ability of vaccine ${ }^{6,7}$ All these interactions involve cellular interactions depending upon patho-physiological changes and induction of immune response. These can be well reflected by the changes in number and types of cells in blood and other blood parameters. These parameters can be easily analyzed. Thus considering all the existing issues and to address the need of hour in light of Indian scenario, the present study was designed to assess the impact of vaccination with live Rev. 1 vaccine and challenge of local virulent strain Brucella melitensis biovar 3 IND1 on various hematological bio markers in pure bred Jamunapari goats.

\section{Material and methods}

\section{Approval of IAEC}

Before the start of study, proposal was submitted to Institutional Animal Ethics Committee (IAEC) of CIRG, Makhdoom. After the approval of respective IAEC, study was conducted in animals as per the guidelines of CPCSEA.

\section{Experimental goats}

Pure bred apparently healthy non pregnant Brucella free Jamunapari adult female goats aged between 2-3years without any history of abortions were selected from Jamunapari Goat shed, ICARCentral Research Institute on Goats (CIRG), Makhdoom, Farha, 
Mathura (India). Selected goats were tested twice at the interval of 28days for Brucella serum antibodies by RBPT; STAT; indirect ELISA and PCR based genus specific amplification from discharges and serum. Three goats found negative twice at the interval of 28days in RBPT, STAT, indirect ELISA and PCR based genus specific amplification were selected and separated for the study.

Place of research work: The study was performed at ICAR-Central Institute for Research on Goats (CIRG), Makhdoom, Farha, Mathura, India. All the selected goats were shifted and maintained in challenge shed of Division of Animal Health, CIRG, Makhdoom, Farha, Mathura (India). All the goats were reared as per the guide lines of CPCSEA with good management practices (GMP) and were provided feed and water ad libitum.

Vaccine: Rev.1 vaccine consisting of Elberg 101 strain was used in study. The Vaccine was procured from Indian Immunological Limited (IIL), Hyderabad.

Challenge strain: Virulent bacterial strain, isolated from stomach content of aborted fetus of Brucella melitensis biovars 3 infected goat was confirmed and submitted to VTCC (Accession no. VTCCBAA228) was used to challenge vaccinated animals. The full genome sequence of the organism has been submitted to NCBI, Data Bank and has been assigned the name of Brucella melitensis biovar 3IND1 (Bmb3IND1).

\section{Vaccination and challenge protocol}

The pure bred apparently healthy non pregnant Brucella free Jamunapari adult female goats $(n=3)$ were vaccinated with standard Rev.1 (IIL, Hyderabad) vaccine as per the manufacturer recommendations. The vaccinated animals were challenged on $28^{\text {th }}$ day post vaccination with live virulent $B$. melitensisbiovar 3 IND1 cultures (109 CFU) through subcutaneous route and monitored for till sacrificed on $90^{\text {th }}$ day of challenge.

Collection of blood samples: The blood was collected before the start of study and during the complete study period on $14^{\text {th }}, 28^{\text {th }}$ day of vaccination and then $14^{\text {th }}, 28^{\text {th }}, 60^{\text {th }}$ and $90^{\text {th }}$ day post challenge. EDTA coated vacutainers (BD Biosciences) were used for the collection of whole blood. The collected samples were immediately transported to laboratory for the analysis of haematological biomarkers.

Haematology of whole blood samples: All the blood samples collected in EDTA coated sterile vaccutainers in goats were subjected to haematological examination with auto analyzer to assess the effect of vaccine on 14 blood parameters including white blood cell (WBC) count, blood lymphocyte, monocytes, granulocytes count and percentage, blood erythrocytes (RBC) count, mean corpuscular volume (MCV), haematocrit (HCT), mean corpuscular haemoglobin $(\mathrm{MCH})$, mean corpuscular haemoglobin concentration (MCHC), $\mathrm{Hb}$ and THR profiles.

Statistical analysis: Various parameters were expressed as mean \pm SE. Mean of parameters at different time intervals after vaccination and challenge were compared using ANOVA. ${ }^{8}$ A value of $\mathrm{P}<0.05$ was considered as statistically significant.

\section{Results}

Whole blood samples collected from experimental goats during study were subjected to assess various blood haematological parameters with the help of auto analyzer within an hour of collection of blood sample. A total 14 parameters were recorded (Table 1), (Figures 1-12).
White blood cell counts reduced significantly in vaccinated animals after vaccination and remained maintained up to $14^{\text {th }}$ after challenge. Then it increased slightly on $28^{\text {th }}$ day in comparison to initial decrease and maintained up to $90^{\text {th }}$ day post challenge. There was no significant difference between post vaccination and post challenge level of white blood cells count (Table 1), (Figure 1). In white blood cells the percentage of lymphocytes significantly increased after vaccination and continues to increase up to $28^{\text {th }}$ day of vaccination. Challenge drastically reduced the lymphocyte percentage $14^{\text {th }}$ day post challenge that recovered to reach at the normal level on $28^{\text {th }}$ day post challenge but continue to increase up to $60^{\text {th }}$ day post challenge making it significantly higher to normal values and finally it comes back to normal values on $90^{\text {th }}$ day post challenge. The lymphocyte percentage at $28^{\text {th }}$ day post challenge was significantly lower to the values on the same day post vaccination (Table 1), (Figure 2). The blood monocytes percentage profile showed significant decline after Rev.1 vaccination. It slightly recovered back after challenge but never reached to its normal values even after $90^{\text {th }}$ day post challenge. There was no significant difference between post vaccination and post challenge vales of monocytes percentages (Table 1), (Figure 3). Granulocytes are suggestive of bacterial infection and vaccination significantly reduced granulocytes percentage up to $28^{\text {th }}$ day post vaccination. The application of virulent challenge strain significantly increased the percentage of granulocytes $14^{\text {th }}$ day post challenge in comparison to healthy animals and vaccinated animals. These values were restored thereafter and reached to normal values on $90^{\text {th }}$ day post challenge (Table 1), (Figure 4). The counting of lymphocyte decreased significantly $14^{\text {th }}$ day post vaccination and almost recovered back to $28^{\text {th }}$ day post vaccination. The challenge with virulent strain again significantly reduced the lymphocyte count in comparison to healthy as well as vaccinated animal. These reduced values showed the trend of increase on $90^{\text {th }}$ day post challenge but the value was significantly lower to healthy animals (Table 1), (Figure 5). Similarly, blood monocytes count significantly reduced $14^{\text {th }}$ day post vaccination and recovered on $28^{\text {th }}$ day post vaccination. The challenge further significantly reduced monocytes in circulation with trend of recovery $28^{\text {th }}$ day post challenge. However, the number of circulating monocytes was significantly lower to the number after vaccination (Table 1), (Figure 6). As expected the total granulocytes count increased significantly after vaccination and challenge. The trend of increase also followed similar trend in both the conditions and even after $90^{\text {th }}$ day of post challenge level was higher in comparison to healthy animals (Table 1), (Figure 7). On vaccination blood RBC count remained almost unaltered showing non-significant difference on vaccination and challenge (Table 1), (Figure 8). Likewise the values of MCV, Hct and $\mathrm{MCH}$ parameters also showed the trend of non significant variation with marginal changes (Table 1), (Figure9-11). $\mathrm{MCHC}$ values increased slightly after vaccination. The reverse trend was observed in challenged animals with reducing values till $60^{\text {th }}$ day of challenge and finally ended with recovery trend on $90^{\text {th }}$ day post challenge (Table 1), (Figure 12). Blood Hb showed almost similar profile in all vaccinated as well as challenged animals and remained almost stable during whole period of study (Table 1). However, challenge slightly reduced the values up to $60^{\text {th }}$ day post challenge. The THR profile of vaccinated and challenged animals showed slight decrease after challenges and recovered $60^{\text {th }}$ day post challenge (Table 1). The blood MPV profile was almost maintained after vaccination but challenged animals showed increasing trend till $90^{\text {th }}$ day post challenge. The values differed non significantly to vaccinated and healthy goats (Table 1). 
Table I Effect of vaccination and challenge on Goat Haematology profile of purebred Jamunapari goats

\begin{tabular}{|c|c|c|c|c|c|c|c|}
\hline \multirow[t]{2}{*}{ Parameters } & \multicolumn{3}{|c|}{ Post Vaccination Schedule } & \multicolumn{4}{|c|}{ Post Challenge Schedule } \\
\hline & 0 day & I4th day & 28th day & I4th day & 28th day & 60th day & 90th day \\
\hline WBC (in millions) & $27.13 \pm 1.73$ & $21.95^{*} \pm 2.44$ & $17.78 * \pm 5.32$ & $17.07 * \pm 2.72$ & $19.32 * \pm 5.27$ & $21.14 * \pm 2.62$ & $19.02 * \pm 3.39$ \\
\hline Lymphocytes (\%) & $33.07 \pm 8.03$ & $39.33 * \pm 0.56$ & $42.77 * \pm 1.78$ & $22.13 * \pm 3.72$ & $31.20 \# \pm 5.25$ & $40.20 * \pm 0.53$ & $32.40 \pm 0.59$ \\
\hline Monocytes (\%) & $3.10 \pm 0.17$ & $2.37 * \pm 0.58$ & $2.33^{*} \pm 0.97$ & $2.50 * \pm 0.71$ & $2.67 * \pm 0.65$ & $2.60 * \pm 0.93$ & $2.63^{*} \pm 0.91$ \\
\hline Granulocytes (\%) & $63.83 \pm 8.11$ & $59.30 \pm 2.04$ & $54.90 * \pm 2.31$ & $75.37 * \# \pm 4.40$ & $66 .|3 \pm 5.6|$ & $56.20 * \pm 0.75$ & $64.97 \pm 1.30$ \\
\hline Lymphocytes (m/mm3) & $11.94 \pm 1.49$ & $8.40 * \pm 0.41$ & $10.00 \pm 1.42$ & $6.53 * \# \pm 1.80$ & $6.12 * \# \pm 0.41$ & $6.60 * \# \pm 0.52$ & $7.35 * \# \pm 0.84$ \\
\hline Monocytes (m/mm3) & $0.75 \pm 0.18$ & $0.52 * \pm 0.15$ & $0.64 \pm 0.13$ & $0.45 * \# \pm 0.18$ & $0.59 * \# \pm 0.10$ & $0.55 * \pm 0.20$ & $0.63 \pm 0.07$ \\
\hline Granulocytes (m/mm3) & II.44 \pm 0.30 & $14.03^{*} \pm 1.18$ & $15.94 * \pm 0.96$ & $13.09 \pm 1.07$ & $\left|4.35^{*} \pm 4.0\right|$ & $15.99 * \pm 2.26$ & $13.52 \pm 2.26$ \\
\hline $\mathrm{RBC}(\mathrm{m} / \mathrm{mm} 3)$ & $9.47 \pm 0.54$ & $9.26 \pm 0.61$ & $9.21 \pm 0.60$ & $8.75 \pm 0.89$ & $8.97 \pm 1.00$ & $10.26 \pm 1.01$ & $9.33 \pm 1.28$ \\
\hline $\mathrm{MCV}(\mathrm{fl})$ & $13.50 \pm 0.40$ & $13.47 \pm 0.44$ & $10.40 * \pm 3.03$ & $13.93 \pm 0.68$ & $14.17 \pm 0.87$ & $14.30 \pm 0.93$ & $14.01 \pm 1.05$ \\
\hline Hct (\%) & $12.67 \pm 0.38$ & $12.37 \pm 0.54$ & $12.63 \pm 1.08$ & $12.13 \pm 1.38$ & $12.63 \pm 1.45$ & $|4.57 \pm| .58$ & $13.47 \pm 1.86$ \\
\hline $\mathrm{MCH}(\mathrm{pg})$ & $5.27 \pm 0.03$ & $5.47 \pm 0.12$ & $5.43 \pm 0.03$ & $5.10 \pm 0.25$ & $4.93 \pm 0.22$ & $5.00 \pm 0.20$ & $5.00 \pm 0.20$ \\
\hline $\mathrm{MCHC}(\mathrm{g} / \mathrm{dl})$ & $39.90 \pm 1.01$ & $41.10 \pm 1.19$ & $40.23 \pm 1.44$ & $37.20 \pm 0.53$ & $35.27 * \pm 0.83$ & $35.63^{*} \pm 1.20$ & $36.10 \pm 1.13$ \\
\hline RDW & $17.43 \pm 0.28$ & $17.40 \pm 0.60$ & $16.90 \pm 0.25$ & $17.43 \pm 0.28$ & $17.93 \pm 0.87$ & $16.97 \pm 0.19$ & $16.40 \pm 0.12$ \\
\hline $\mathrm{Hb}(\mathrm{g} / \mathrm{dl})$ & $5.07 \pm 0.26$ & $5.10 \pm 0.36$ & $5.07 \pm 0.35$ & $4.53 \pm 0.58$ & $4.47 \pm 0.57$ & $5.20 \pm 0.60$ & $4.73 \pm 0.74$ \\
\hline THR (m/mm3) & $278.00 \pm 48.39$ & $278.67 \pm 51.85$ & $275.33 \pm 68.56$ & $249.67 \pm 61.17$ & $244.33 \pm 69.49$ & $259.67 \pm 87.19$ & $241.67 \pm 80.43$ \\
\hline MPV (fl) & $5.70 \pm 0.15$ & $5.67 \pm 0.15$ & $5.70 \pm 0.26$ & $5.73 \pm 0.23$ & $5.73 \pm 0.22$ & $5.73 \pm 0.29$ & $5.80 \pm 0.29$ \\
\hline Pct (\%) & $0.16 \pm 0.02$ & $0.16 \pm 0.02$ & $0.15 \pm 0.03$ & $0.14 \pm 0.03$ & $0.14 \pm 0.03$ & $0.14 \pm 0.04$ & $0.13 \pm 0.04$ \\
\hline PDW & $3.53 \pm 0.19$ & $3.70 \pm 0.38$ & $3.73 \pm 0.71$ & $3.57 \pm 0.58$ & $3.47 \pm 0.52$ & $3.77 \pm 0.82$ & $3.53 \pm 0.76$ \\
\hline
\end{tabular}

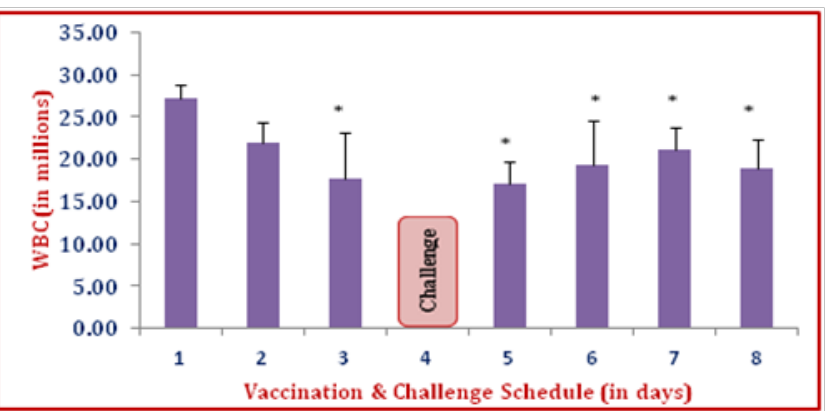

Figure I White blood cells (WBC) profile.

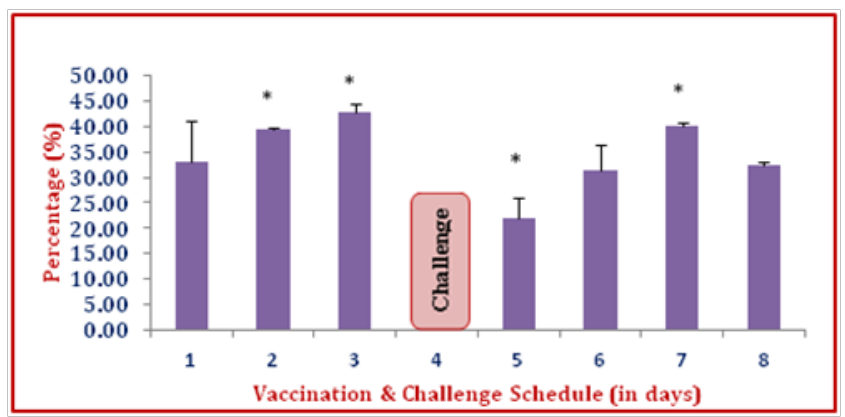

Figure 2 Lymphocyte percentage profile.

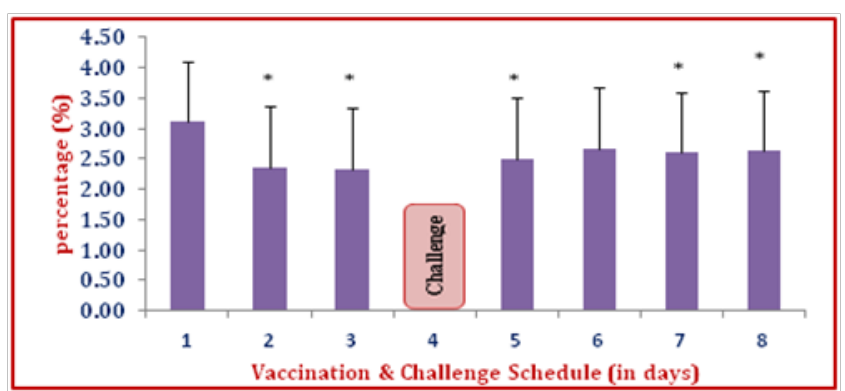

Figure 3 Monocytes percentage profile.

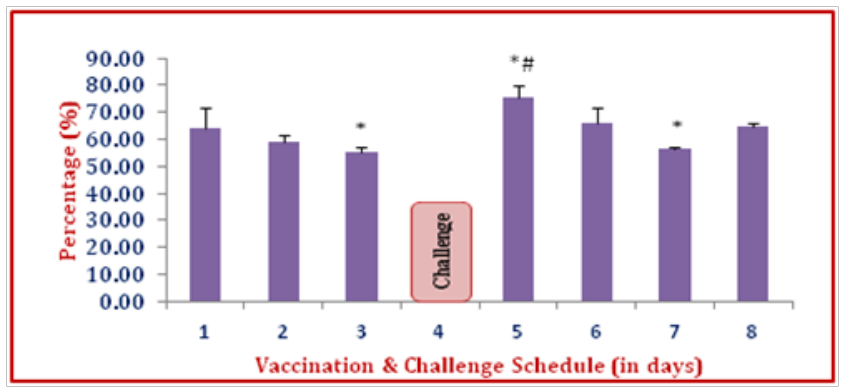

Figure 4 Granulocytes percentage profile.

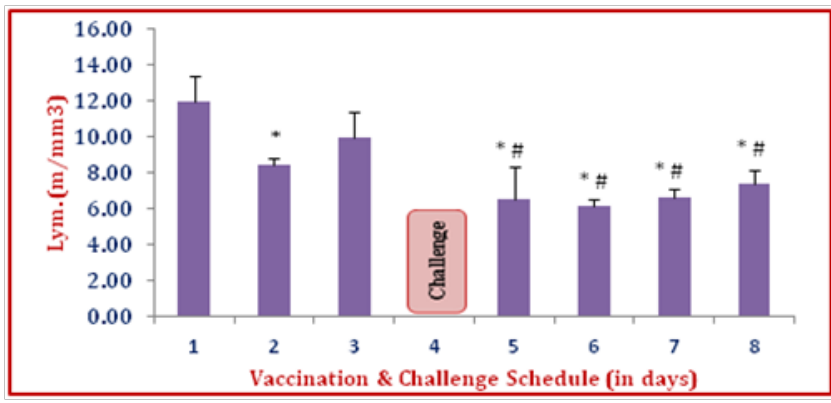

Figure 5 Lymphocytes profile.

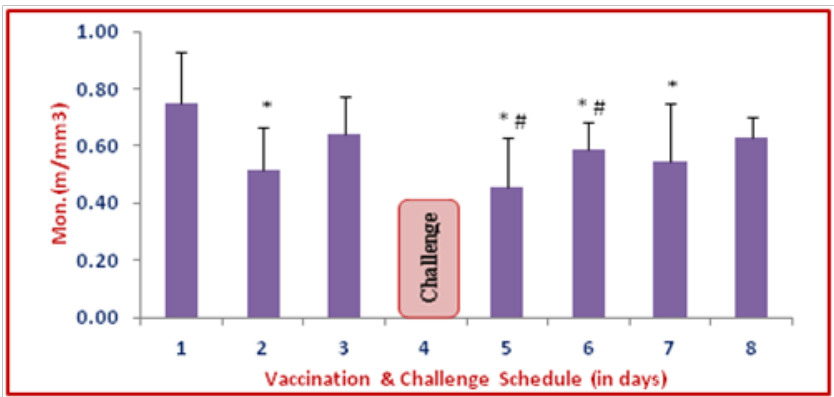

Figure 6 Monocytes profile.

Citation: Kumar A, Gupta VK,Verma AK, et al.Assessment of hematological bio markers during vaccination and challenge of brucella melitensisin goats. Int J Vaccines Vaccin. 2017;4(2):I I-12. DOI: 10.15406/ijvv.2017.04.00079 


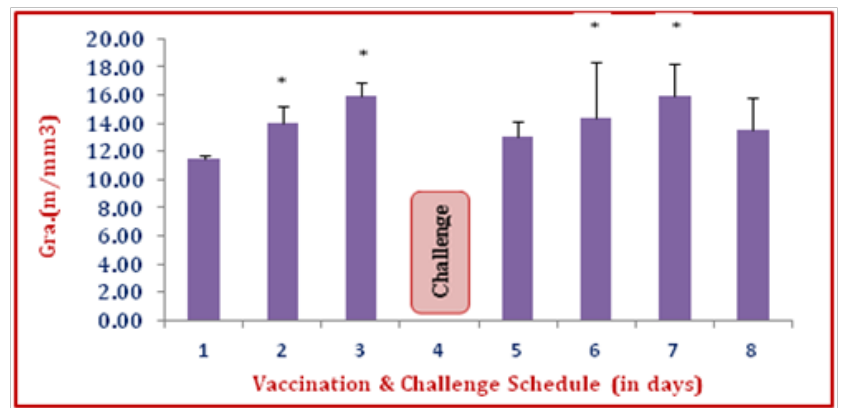

Figure 7 Granulocytes profile.

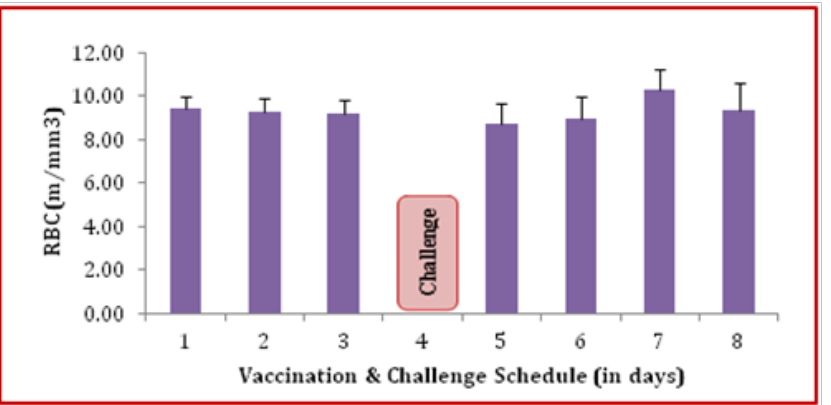

Figure 8 Blood RBC profile.

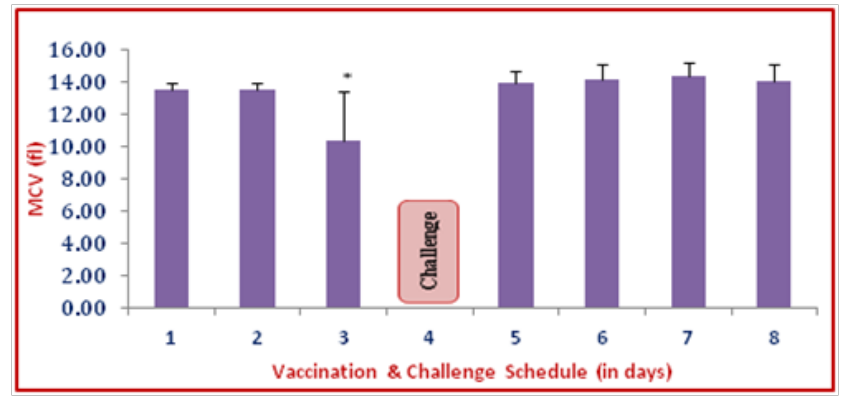

Figure 9 Blood MCV profile.

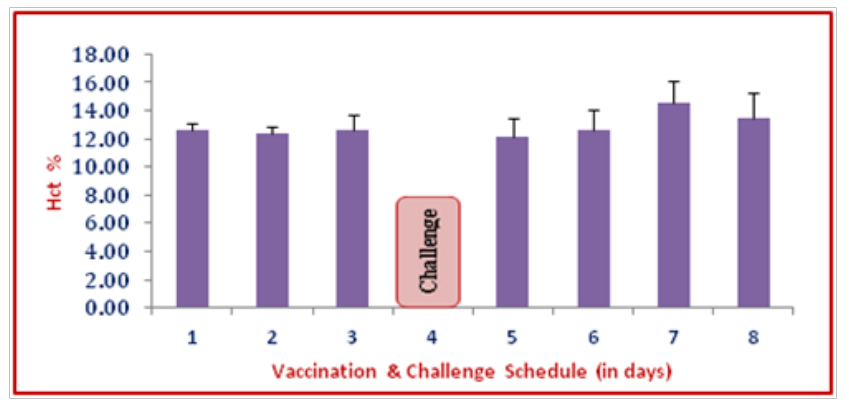

Figure 10 Blood Haematocrit profile.

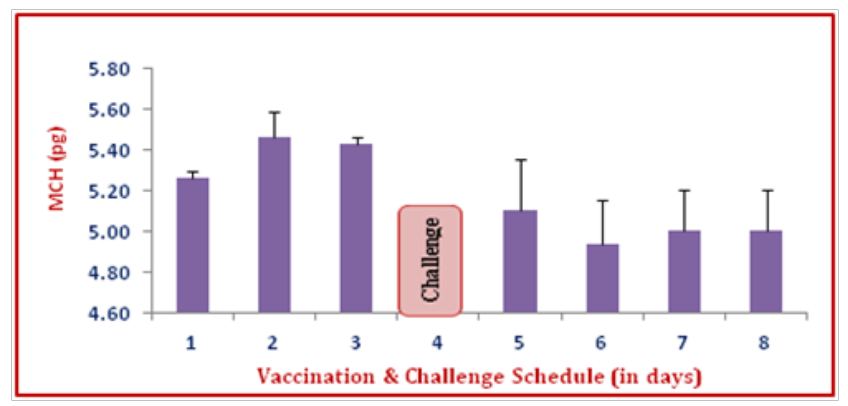

Figure II Blood $\mathrm{MCH}$ profile.

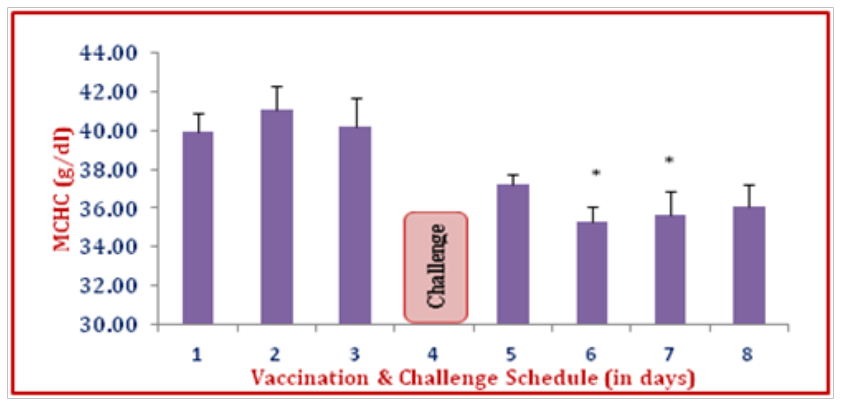

Figure 12 Blood MCHC profile.

\section{Discussion}

The protective efficacy of a vaccine greatly depends on the Brucella virulence and challenge dose. In this regard, based on a substantial data dedicated to standardize the mouse model for vaccine testing, a standardized protocol for controlling the quality of $B$. melitensis Rev.1 vaccines has been accepted by the World Organization for Animal Health. ${ }^{9}$ The vaccine efficacy is generally analyzed 2 weeks after challenge, when the differences between the reference-vaccinated and unvaccinated controls have reached at maximum. ${ }^{7}$ Considering these facts, goats were challenged $28^{\text {th }}$ day post vaccination and sacrificed $90^{\text {th }}$ day post challenge. In protection studies use of homologous challenge strain is recommended. ${ }^{6,9,10}$ However the protection against Brucella is genus-specific and not speciesspecific. ${ }^{6}$ Based on these recommendations, B. melitensis biovar3 IND1, the local virulent strain, was used to challenge vaccinated animals. On $28^{\text {th }}$ day post vaccination, animals were challenged with live virulent Bmb3IND1 cultures (109 CFU) through sub cutaneous route. Brucella is facultative intracellular parasite of reticuloendothelial system. After getting entry into animal body, Brucella are phagocytosed by lymphoepithelial cells of gut-associated lymphoid tissue, reach nearest lymph nodes and ultimately localize in lymph nodes, spleen, mammary glands, joints, kidneys and bone marrow, being carried by the macrophages. ${ }^{11}$ During this process, organisms also come into circulation for a brief duration so assessment of haematological parameters can be of use as these are easy to perform, cheaper and can be performed in short time. In present study 14 various hematological parameters were performed, out of these parameters blood erythrocytes (RBC) count, mean corpuscular volume (MCV), haematocrit $(\mathrm{HCT}), \mathrm{Hb}, \mathrm{THR}$, and mean platelet volume (MPV) profiles showed nonsignificant variation in healthy, vaccinated and challenged goats. Thus these parameters of no use in protection as well as challenge studies in particular to brucellosis in goats. The challenge reduced the values of mean corpuscular haemoglobin $(\mathrm{MCH})$ and mean corpuscular haemoglobin concentration (MCHC) reduced after challenge in comparison to healthy goats. Out of other parameters, Brucella being intra cellular bacteria cells like lymphocytes, granulocytes and monocytes count and percentage in circulation are of prime importance. The neutrophils are probably the first immune associated cells in the host defense to encounter Brucella. Rapid phagocytosis of virulent and attenuated Brucella strains by neutrophils occurs only after opsonization with normal serum, ${ }^{12}$ suggesting that opsonization is a prerequisite for phagocytosis. However, the survival of Brucella in neutrophils during early infection has been observed, ${ }^{13}$ suggesting that the transportation of Brucella to lymphoid tissues can be mediated by neutrophils. ${ }^{14}$ Moreover, monocytic membrane-bound thrombomodulin (TM), a component in CD14/TLR4/MD-2 complex participates in the LPS- 
and Gram-negative bacteria-induced inflammatory response. ${ }^{15}$ The inhibition of bacterial dissemination with a deficiency of myeloid TM may be caused by the early increase in neutrophil infiltration. Later on it enters into macrophages and requires cellular immune response for the clearance of Brucella. ${ }^{15}$ For this clearance, the main role is played by lymphocytes so their number in circulation also play crucial role. ${ }^{16}$ Appreciable granulocytes in the haematological profile are suggestive of bacterial infection. Both vaccine and challenge strain being live, increase in granulocyte number and percentage immediately after vaccination and challenge in particularly on $14^{\text {th }}$ day were suggestive of good interaction of brucella in circulation and play crucial role in humoral immune response. This increased granulocyte count is expected to transport Brucella to lymphoid tissues. ${ }^{14}$ and thus with the internalization of Brucella organism, cytotoxic $\mathrm{T}$ cells become activated increasing proliferation of lymphocyte and finally leading to cell mediated immune response. The higher values of granulocyte in challenged animals indicated more virulence of organisms. The lymphocyte/granulocyte proportions in blood vary according to animal species and breed and may range in between $80 / 15 \%$ to $90 / 9$ $\%$ on Brucella infection. ${ }^{7}$ It has been suggested that a phenotype may be related to Brucella clearance due to the regulatory action which neutrophils can display over macrophages. ${ }^{17}$ The increase of lymphocytes percentage in total white blood cell count is suggestive of enhanced cellular response after vaccination which is a requisite to avoid its colonization and thus, to overcome Brucella infection. ${ }^{7}$ In brucellosis, APCs such as monocytes play major roles as sentinels for first line alerts or as mediators that shape the adaptive immune response..$^{18}$ For clearance of brucella number of monocytes increased in circulation but overall percentage in total cell count remained almost un altered as clearance involved the engulfment and clearance of infected lymphocytes from circulation as well as from tissues. The challenge with virulent culture showed initial rise in bacterial count in circulation attracting granulocytes as first line of defense but internalization of organism insisted increase in lymphocyte count.

\section{Conclusion}

The present study revealed that out of commonly used fourteen hematological parameters only few revealed its significant role in protection as well as challenge studies performed in Pure bred indigenous Jamunapari Goats. The percentage of lymphocytes and granulocytes in vaccination with live Rev.1 vaccine does not have any conclusive assessment. It is only the count that can be used to draw any inference. The blood level of granulocytes, monocytes and lymphocytes in number can only be correlated with the impact of vaccine as well as challenge with virulent strain, thus can be used as hematological biomarkers.

\section{Acknowledgments}

None.

\section{Conflicts of interest}

Author declares there are no conflicts of interest.

\section{Funding}

None.

\section{References}

1. Singh BB, Dhand NK, Gill JP. Economic losses occurring due to brucellosis in Indian livestock populations. Prev Vet Med. 2015;119(3-4):211-215.

2. Kumar A, Gupta VK, Verma AK, et al. Vaccines for Caprine Brucellosis: Status and Prospective. Int J Vaccines Vaccin. 2016;2(3):00030.

3. Singh SV, Singh N, Shankar H, et al. Seroprevalence of brucellosis in a few important Indian goat breeds. Small Rumi Res. 1998;30(2):93-98.

4. Manual of standards for diagnostic tests and vaccines (3rd edn), Office International des Epizooties, France. 2010. P.1-251.

5. WHO / MZCP. Human and Animal Brucellosis. Report of a WHO MZCP workshop. Damascus, Syrian Arab Republic. 1998.

6. Bosseray N, Plommet M, De Rycke J. Evolution de l'infection de la souris par Brucella abortus, Brucella melitensis et Brucella suis vers l'état chronique et la guérison. Ann Rech Vet. 1982;13(2):153-161.

7. Grillo MJ, Blasco, JM, Gorvel, et al. What have we learned from brucellosis in the mouse model? Vet Res. 2012;43:29.

8. Snedecor GW, Cochran WG. Statistical methods. (7th edn), Oxford and IBH Publishing company, India. 1989.

9. Office International des Epizooties (OIE). Caprine and bovine brucellosis (excluding Brucella ovis). In: Manual of Diagnostic Tests and Vaccines for Terrestrial Animals, (5th edn), Paris. 2008.

10. González D, Grilló MJ, De Miguel MJ, et al. Brucellosis vaccines: assessment of Brucella melitensis lipopolysaccharide rough mutants defective in core and O-Polysaccharide synthesis and export. PLoS ONE. 2008;3(7):e2760.

11. Pizarro-Cerda J, Meresse S, Parton RG, et al. Brucella abortus transits through the autophagic pathway and replicates in the endoplasmic reticulum of nonprofessional phagocytes. Infect Immun. 1998;66(12):5711-5724

12. Young EJ, Borchert M, Kretzer FL, et al. Phagocytosis and killing of Brucella by human polymorphonuclear leukocytes. $J$ Infect Dis. 1985;151(4):682-690.

13. Riley LK, Robertson DC. Ingestion and intracellular survival of Brucella abortus in human and bovine polymorphonuclear leukocytes. Infect Immun. 1984;46(1):224-230.

14. Nathan C, Shiloh MU. Reactive oxygen and nitrogen intermediates in the relationship between mammalian hosts and microbial pathogens. Proc Natl Acad Sci USA. 2000;97(16):8841-8848.

15. Ma CY, Shi GY, Shi CS, et al. Monocytic Thrombomodulin Triggers LPS- and Gram-Negative Bacteria-Induced Inflammatory Response. The J Immuno. 2012;188(12):6328-6337.

16. Singh A, Gupta VK, Kumar A, et al. 16S rRNA and Omp31gene based molecular characterization of field strains of $B$. melitensis from aborted foetus of goats in India. ScientificWorldJournal. 2013;2013:160376.

17. Martirosyan A, Moreno E, Gorvel JP. An evolutionary strategy for a stealthy intracellular Brucella pathogen. Immunol Rev 2011;240(1):211-234.

18. Hoebe K, Janssen E, Beutler B. The interface between innate and adaptive immunity. Nat Immunol. 2004;5(10):971-974. 